\title{
Contextual pre-conditioning and sustained conditioning of cells dictate cell-target therapeutic outcome in tumor cell apoptosis/anti-apoptosis
}

\begin{abstract}
Significant participation of constitutive self-programming of apoptotic pathways indicates an in-built series of mechanistic pathways that protect against susceptibility patterns of evolving carcinogenesis. As such the incremental exposure pathways include dimensional re-formulation of events as well-established programmed schemes of network operability within the further conceptual realization of direct and indirect effects on cell viability once malignant transformation has taken place. Specific contexts of strict pre-conditioning and further stages of sustained conditions of cells such as seen in epithelial cells of breast and uterus come to operate in dominant fashion in determining cell-subset fate outcome when such cells are exposed to stress or injury. Chemotherapeutic resistance of malignant tumor cells would arise in terms of such contextual conditioning that includes in particular the genotype of the cells concerned. Mechanistic individualization of component intermediates of specific pathways is modulated in a manner that enhances such context of involvement of integral cells beyond the simple conformations of even interactive systems of effect and progression. Initial response of tumor cells to administered chemotherapeutic agents is supplemented by the development of second-stage resistance to such agents in many patients and would be indicative of adaptive low-metabolic status and non-proliferation of the tumor cells. In this setting, parameters of integration belie stratified re-organization of individual pathway mechanics and would implicate otherwise homeostatic counter-systems that enhance pro-apoptosis or anti-apoptosis in response to radiation or chemotherapy of tumor cells.
\end{abstract}

Keywords: carcinogenesis, malignant, pre-conditioning, conditions, injury, tumor cells, progression, chemotherapeutic, patients, chemotherapy; low-metabolic, proliferation, mechanistic, contextual

\author{
Volume 2 Issue 3 - 2017
}

\author{
Lawrence M Agius \\ Department of Pathology, University of Malta Medical School, \\ Europe
}

Correspondence: Lawrence M Agius, Department of Pathology, Mater Dei Hospital, Tal-Qroqq, University of Malta Medical School, 27 "Ballarat", Guzeppe Caruana Street, Msida, Tal-Virtu, Rabat, Rbt09, Malta, Europe, Tel 3562 I451752, Email lawrence.agius@um.edu.mt

Received: October 19, 2016 | Published: February 28, 2017

\section{Introduction}

Apoptosis is consistently associated with effects also of mechanisms that promote anti-apoptosis via systems that modulate especially the activity of master transcriptional factors such as c-Myc, Nuclear Factor-kappaB (NF-kB), E2F-1, p53 and Retinoblastoma gene (RB). Loss of wild-type p53 activity is thought to be a major predictor of failure to respond to chemotherapy in a wide variety of malignant neoplasms. ${ }^{1}$ It is increasingly recognised that different neoplasm types display different degrees of addiction to individual Bcl-2 family proteins in particular. $^{2}$

Systems of progression of cancer cells are especially prone to the secondary development of resistance to chemotherapeutic agents, and such resistance arises from failure of apoptotic mechanisms in response to chemotherapy-induced forms of stress or injury to the malignant cells. Development of a vector or vehicle that can selectively and efficiently deliver the gene to target cells with minimal toxicity is urgently needed, as with phosphate and tennis homolog and tumor necrosis factor-related apoptosis-inducing ligand. ${ }^{3}$ Programmed Cell Death can significantly sensitize different cancers to chemotherapy by interaction with the p53 pathway to induce cell apoptosis. ${ }^{4}$

System parameter outline enforce the evolutionary dimensions within secondary pathways of influence in pro-apoptosis. Checkpoint kinases 1 and 2 are critical regulators of the DNA damage response, and may permit recovery from therapy-associated genomic stress. ${ }^{5}$ In such manner, the consequential sequence of network evolution further compromise pro-apoptosis within the framework viability background as dictated by such network dysfunctionality. Relapses or primary refractory disease in tumors such as non-Hodgkin lymphoma are commonly observed and associated with dismal prognosis. ${ }^{6}$ It is further to the characterisation and re-characterization of formulae in evolutionary exposure of cells to carcinogenic agents that there also emerges the profile dimensions of an either/or outcome product for possible malignant transformation. In such manner, the global dimensionality of evolutionary outcome in malignant transformation includes the prior conditioning and the delayed effects of evolving pro-apoptosis. Targeted therapeutics facilitate site-specific delivery with minimum off-target effects and include nucleic-acid based aptamers that are of small size, and have long shelf-life and ease in synthesis. ${ }^{7}$ It is significant to view the progressive nature of carcinogenesis in terms of the consequent development and further modulation of induction pathways that strictly formulate the integral malignant transformation of cells both individually and as community networks. Lovastatin treatment for glioblastoma could inhibit NF-kappaB and Erk/MAPK pathways but activate JNK pathway; it sensitises TRAIL-induced apoptosis by up-regulation of DR5 level via NF-kappaB inactivation. ${ }^{8}$

The consequentiality of such proposed formulae for further carcinogenic steps in evolution will hence conform to the further promo- 
tional deviation of genetic lesions as well-illustrated by loss of tumor suppressor functionality of such gene products as p53. It is through the increasing effectiveness of substantial deviation from gene suppressive action in carcinogenesis that there would emerge the schematic reconstitution of pathway events as maladaptive response to DNA damage and of stress induction of the affected cells. Liposome nanoparticle deliver of miR-101 and doxorubicin simultaneously may result in significant synergetic antitumor effects in hepatocellular carcinoma. $^{9}$

In particular, it is a central premise that a dual series of pathways of apoptosis and of anti-apoptosis operates to control fundamental outcomes of cell death or of cell survival. Increasing evidence indicates that basic resistance of small-cell lung cancer to chemotherapy and radiation depends on altered balance in apoptosis/anti-apoptosis. ${ }^{10}$ Pro-apoptosis versus pro-survival mechanisms are considered, however, to interact involving such agonists as c-Myc, NF-kB, and various different members of the Bcl-2 family of factors. Nitric oxide mediates inhibition of NF-kappaB and of its downstream anti-apoptotic gene targets and so sensitizes tumor cells to apoptosis as induced by chemotherapeutic drugs. ${ }^{11}$ In the case of caspases such as caspase 8, allowing interactions between the intrinsic and extrinsic pathways and leading to apoptosis, a complex interplay of pathways operates within the mechanistic progression of cell-death promoting factors. This determines inherent progression of pro-apoptosis, thus undoubtedly promoting susceptibility to possible modulation by various agonists or stress-inducing events.

\section{Reactive oxygen species}

Oxidative free-oxygen radicals have been shown to participate in ongoing events inducing the activation of transcription and also translation of proteins involved in both apoptosis and anti-apoptosis. DNA repair, DNA synthesis, cell adhesion, cell cycle regulation, mitotic spindle checkpoint may be upregulated by novel platinum complexes and so trigger inherent defense cell mechanisms and potential resistance to apoptosis. ${ }^{12}$

In fact, an emerging concept of initial stimulation of anti-apoptotic mechanisms recognizes a status formulation that precedes the activation of stress-induced pro-apoptotic pathways in a manner that specifically redefines the nature of complexity of cell outcome in response to various injuries, including chemotherapeutic exposure of malignant neoplasms.

Thus, a respiratory increase in activity of mitochondria and the increased production of ATP (Adenosine triphosphate) are a system pathway response in their own right in formally defining the nature of cellular response to injury in a manner specifically inducing initial anti-apoptotic protection of the cells affected by stress. Photodynamic therapy utilizing an $18 \mathrm{kDa}$ mitochondrial translocator protein target by administering IR700DX-6T induced apoptosis in MDA-MB-231 breast cancer cells. ${ }^{13}$

Indeed, growth factor withdrawal, such as insufficient supply of Nerve Growth Factor to pheochromocytoma-derived cell line, constitutes a real form of stress/injury that is reflected in pro-apoptotic responses. Such conceptual idealization is indicative of a central integrative functionality of injured cells that is delineated within the profile mechanisms of balance or of disturbed homeostatic systems of intrinsic and extrinsic pro-apoptosis. Mammalian target of rapamycin, (mTOR) downstream of the phosphatidylinositol 3-kinase signaling pathway, regulates transcription, translation, proliferation, apoptosis and autophagy; mTOR acquires transforming activity through genetic changes in tumors. ${ }^{14}$

\section{Pro-survival}

Derivative dimensions of pro-survival are inductive formulas in mediating inherently constitutive pathways of control of possible cell outcome that depend particularly on the over-expression of various modulator proteins. In such mode, the over-expression of $\mathrm{Bcl}-2$ is important in determining promotion of anti-apoptotic effects when activated by cell stress. Indeed, Bcl-2 was initially identified as a constant over-expression of the protein in lymph nodes transformed by follicular lymphoma.

Hence, also, delivery of mediator proteins in apoptosis-related systems comes to play an important role in determining cell fate, including especially NF-kB (Nuclear Factor-kappaB) translocation into the nucleus subsequent to inhibition/degradation of inhibitors of NF-kB in the cytosol. Also, the migration of Akt, a central pro-survival agonist, to the cell membrane is formulated in a manner that modulates its effects. The Mdm2 in the cytoplasm may translocate to the nucleus where it can inhibit p53 action and thus promote anti-apoptosis.

The important aspects of operability of the apoptotic mechanisms include the active, energy-utilizing demands of such systems in promoting caspase activation and progression and in the degradation of cell structural components. Enantiomeric CopA3 dimer peptide is a coprisin analog and this induces apoptosis and necrosis of gastric carcinoma cells in vitro and in vivo by mediating interactions with phosphatidylserine and phosphatidylcholine. ${ }^{15}$

It is further to the availability of energy-rich substrates that apoptosis is less efficient in cells with low metabolic activity. Malignant tumor cells that are not proliferating and non-active metabolically are much less susceptible to apoptotic cell death as potentially induced by chemotherapeutic agents. This is a major challenge that often or usually limits severe therapeutic outcome to chemotherapeutic agent administration in patients with malignancy. Exosomes are effective in inducing drug resistance to chemotherapy and delivery of microRNA-222 via and thus may constitute a possible mechanism. ${ }^{16}$

\section{Apex operants}

Apex pathway mechanisms include especially the effects of free oxygen radicals on master genes such as c-Myc. It is interesting to note, in this regard, that the possible outcome with c-Myc activation and transcription includes apoptosis concurrent with the induced onset also of cell proliferation by c-Myc protein.

MicroRNA-92a promotes tumor growth and metastasis, and also induces chemoresistance in non-small cell lung carcinoma by downregulating phosphatase and tensin homolog (PTEN). ${ }^{17}$ The indicative context of apoptosis and anti-apoptosis and the timing and sequence of induced effects by various different converging stimuli allow a permissive series of stabilization and de-stabilization effects that determine, in large measure, whether apoptosis or anti-apoptosis proves the dominant cellular outcome.

In this regard, the potentiality for survival of malignant cells exposed to radiation or to chemotherapeutic agents depends on a basic duality of apoptosis versus anti-apoptosis that in turn is integral constitution of such contextual conditioning of the cells affected. Silenc- 
ing expression of fibroblast growth factor receptor 4 could increase apoptosis rate and decrease proliferation and invasion by gastric carcinoma cells both in vitro and in vivo. ${ }^{18}$

\section{Degradation of proteins}

The ubiquitin-proteasomal pathway and various activities such as calcium influx within cells or from the endoplasmic reticulum are powerful agonist pathways that delineate component effects of membrane and ion-channel participation within systems determining potential cell outcome to stress or injury. The participation of soluble factors such as lipocalins can deliver agonists or lipophilic agents to cell membrane receptors in a manner that may be further amplified within the cytoplasm of the cells. The intriguing integrative roles of cell components belies a basic linear organization of the caspase systems in a manner that further modifies the true nature of conducive and interactive profiles of activity of apoptotic mechanisms. It is possible that more than one death program may be necessary to ensure cell death fidelity. ${ }^{19}$

It is relevant to view the incremental effect of various anti-apoptotic pathways in terms of formulated dimensional modulations determined especially by the genotype of the affected cells. Hence, concurrent events of pro-apoptosis and of anti-apoptosis allow permissive conditions of incremental susceptibility for one or other pathway in determining final cell death or survival. Glioma stem cells are believed to promote relapse and chemoresistance of brain tumors; metformin and temozolomide act synergistically to inhibit proliferation and induce apoptosis in both glioma cells and glioma stem cells, with enhanced reduction of mTOR, 4EBP1, and S6K phosphorylation. ${ }^{20}$

Ubiquitin-binding and proteasomal degradation of proteins involved in either apoptosis or anti-apoptosis depends on the active degradation of chaperones and co-chaperones within the cytosol, including 4-3-3 protein. Phosphorylation status of various intermediates within these dual counter-pathways also is an important system complex series of mechanisms that determines such component agonists as Akt and also inhibitors of NF-kB. In this way, kinases and phosphatases such as PTEN are instrumental within the integrative dimensions of cell response to injurious agents. Besides acting as a crucial mediator of apoptosis, caspase 3 in dying tumor cells may drive proangiogenic response after irradiation..$^{21}$

\section{Early-stage cell stress}

The early stages of cell stress are characterized by augmentation of anti-apoptotic effects in modes of attempted pro-survival of the cells. The apoptosis mechanisms, although strictly energy-dependent, can also be viewed as de-functional or de-fault states that depend also on mechanistic inadequacy of anti-apoptosis. This is consistent, for example, with the early generation of oxidative intermediates such as free oxygen radicals and of apo-cytochrome $\mathrm{c}$ and also the degree of counter-activity of cytochrome oxidase dismutases such as $\mathrm{Cu} / \mathrm{Zn}$ SOD and Mn-SOD

Cytochrome c release from the mitochondria is a particularly illustrative example of the inherent nature of counter-mechanisms in apoptosis/anti-apoptosis. The ability of Bcl-2 and $\mathrm{Bcl}-\mathrm{xL}$ in controlling the membrane transition pore and the maintenance of the mitochondrial membrane polarization is counter-acted by other members of the Bcl2 family of factors such as Bax, Bid and Bad. Inherent homeostasis in cell-fate determination is reflected also in both potential pro-apoptosis and anti-apoptosis outcome in the generation of excess oxidative free radicals. On the other hand, peptides are useful therapeutic agents in cancer due to their high target selectivity and exceptional ability to penetrate cell membranes. ${ }^{22}$ Antigen spreading-induced CD8+T cells are capable of eliminating malignant mesothelioma cells and of inducing apoptosis of myeloid-derived suppressor cells. ${ }^{23}$

Hence, amplification progression is a consequence of convergence of counter-mechanisms within single integral components of apoptosis and anti-apoptosis; this helps explain the effects of c-Myc in both inducing cell proliferation and also in enhancing apoptosis of the same cells. Also, glioblastoma cells involve dual Epidermal/GSTP1 in inducing functional inhibition of JNK signaling, with significant suppression of both spontaneous and drug-induced apoptosis in tumor cells. ${ }^{24}$ The stabilization or destabilization of $\mathrm{p} 53$ illustrates potent mechanisms that are further propagated in terms of the enhanced pro-apoptotic actions of p 53 versus an inherent tendency for increased destabilization as induced by Akt.

\section{Concluding remarks}

Pro-caspase auto-cleavage and enzymatically-induced cleavage of these proteolytic enzymes have traditionally been considered the central players in activating apoptosis in terms of operatively independent duality of intrinsic and extrinsic pathways, in turn further comprising both executive and initiator caspases.

The placement operability of a number of master genes tends to re-define duality of apoptosis/anti-apoptosis as cooperative systems in ways that de-function cell pathways such as the binding to various promoter regions of major transcription factors. Highly conserved members of both pro-apoptotic and anti-apoptotic pathways are inherently less specific in outcome determination than has been traditionally believed. Contextual conditioning of affected cells and cell genotype are integrating mechanisms in their own right in delineating final cell fate outcome. Such cell conditioning and pre-conditioning are instrumental in programming the mechanistic evolution of dual counter-pathways in a manner that specifically helps in the realization of integrative opposing systems.

Malignant transformation of cells may analogously parallel such counter-mechanisms that are less amenable to definable mechanistic operability of individual pathways. In a real sense, integration of pro-apoptosis and anti-apoptosis would emerge as inherent constitutive cellular systems that promote attributes of pre-conditions as contextually dictated by over-expression of protein mediator proteins and specific genotype, as seen especially in epithelial cells of breast and uterus.

\section{Acknowledgements}

None.

\section{Conflict of interest}

Author declares that there is no conflict of interest.

\section{References}

1. Cappadone C, Stefanelli C, Malucelli E, et al. p53-dependent and p53-independent anticancer activity of a new indole derivative in human osteosarcoma cells. Biochem Biophys Res Commun. 2015;467(2):348-353. 
2. Bose P, Gandhi V, Konopleva M. Pathways and mechanisms of venetoclax resistance. Leuk Lymphoma. 2017;31:1-17.

3. El Sharkawi FZ, Ewais SM, Fahmy RH, et al. PTEN and TRAIL genesloaded zein nanoparticles as potential therapy for heptocelluar carcinoma. J Drug Target. 2017;31:1-29.

4. Zhuge C, Sun X, Chen Y, et al. PDCD5 functions as a regulator of p53 dynamics in the DNA damage response. J Theor Biol. 2015;388:1-10.

5. Zeng L, Beggs RR, Cooper TS, et al. Combining Chk1/2 inhibition with cetuximab and radiation enhances in vitro and in vivo cytotoxicity in head and neck squamous cell carcinoma. Mol Cancer Ther. 2017;16(4):591-600

6. Bhatt S, Parvin S, Zhang Y, et al. Anti-CD20-Interleukin-21 fusokine targets malignant B-cells via direct apoptosis and NK-cell dependent cytotoxicity. Blood. 2017;129(16):2246-2256.

7. Chaudhry S, Kanwar RK, Kanwar JR. Targeted theranostics against solid cancer using metal bond milk protein and aptamers. Curr Top Med Chem. 2017;17(18):2100-2111.

8. Liu PC, Lu G, Deng Y, et al. Inhibition of NF-kappaB pathway and modulation of MAPK singling pathways in glioblastoma and implications for Lovastatin and tumor necrosis factor-related apoptosis inducing ligand (TRAIL) combination therapy. PLoS One. 2016;12(1):e0171157.

9. Xu F, Liao JZ, Xiang GY, et al. MiR-101 and doxorubicin codelivered by liposomes suppressing malignant properties of hepatocellular carcinoma. Cancer Med. 2017;6(3):651-661.

10. Loot Y, Calderaro J, Soria JC, et al. Anti-apoptotic mechanisms in smallcell lung carcinoma. Ann Pathol. 2010;30(1):17-24.

11. Bonavida B, Garban H. Nitric oxide-mediated sensitization of resistant tumor cells to apoptosis by chemo-immunotherapeutics. Redox Biol. $2015 ; 6: 487-494$

12. Pang SK, Yu CW, Guan H, et al. Impact of oxaliplatin and a novel DACHplatinum complex in the gene expression of HCT116 colon cancer cells Oncol Rep. 2008;20(5):1269-1276.

13. Zhang S, Yang L, Ling $X$, et al. Tumor mitochondria-targeted photodynamic therapy with a translocator protein (TSPO)-specific phtosensitizer. Acta Biomater. 2015;28:160-170.
14. Yamaguchi H, Kawazu M, Yasuda T, et al. Transforming somatic mutations of mTOR kinase in human cancer. Cancer Sci. 2015;106(2):1687-1692.

15. Lee JH, Kim IW, Shin YP, et al. Enantiomeric CopA3 dimer peptide suppresses cell viability and tumor xenograft growth of human gastric cancer cells. Tumor Biol. 2015;37(3):3237-3245.

16. Yu DD, Wu Y, Zhang XH, et al. Exosomes frome adriamycin-resistant breast cancer cells transmit drug resistance partly by delivering miR-222. Tumor Biol. 2015;37(3):3227-3235.

17. Ren P, Gong F, Zhang Y, et al. MicroRNA-92a promotes growth, metastasis, and chemoresistance in non-small cell lung cancer cells by targeting PTEN. Tumour Biol. 2015;37(3):3215-3225.

18. Ye Y, Jiang D, Li J, et al. Silencing of FGFR4 could influence the biological features of gastric cancer cells and its therapeutic value in gastric cancer. Tumour Biol. 2015;37(3):185-195

19. Malin JZ, Shaham S. Cell death in C. elegans development. Curr Top Dev Biol. 2015;114:1-42.

20. Yu Z, Zhao G, Xie G, et al. Metformin and temozolomide act synergistically to inhibit growth of glioma cells and glioma stem cells in vitro and in vivo. Oncotarget. 2015;6(32):32930-32943.

21. Feng X, Tian L, Zhang Z, et al. Caspase 3 in dying tumor cells mediates post-irradiation angiogenesis. Oncotarget. 2015;6(32):32353-32367.

22. Kim S, Lee JH, Kang I, et al. An amphiphilic peptide induces apoptosis through the miR29b-p53 pathway in cancer cells". Mol There Nuclei Acids. 2016;5(7):e330.

23. Yu Z, Tan Z, Lee BK, et al. Antigen spreading-induced CD8+T cells confer protection against the lethal challenge of wild-type malignant mesothelioma by eliminating myeloid-derived suppressor cells. Oncotarget. 2015;6(32):32426-32438.

24. Okamura T, Antoun G, Keir ST, et al. Phosphorylation of GSTP1 by EGFR promotes formation of the inhibitory GSTP1-JNK complex and suppresses JNK downstream signaling and apoptosis in brain tumor cells. J Biol Chem. 2015;290(52):30866-30878. 